\title{
CAPACIDAD DE CODIFICAR Y DECODIFICAR EMOCIONES FACIALES EN ESTUDIANTES UNIVERSITARIOS DE INGENIERÍA
}

\section{ABILITY TO ENCODE AND DECODE FACIAL EMOTIONS IN ENGINEERING COLLEGE STUDENTS}

\author{
${ }^{1}$ Mario Buenaventura Castillo Hilario
}

\begin{abstract}
Resumen
Esta investigación se propuso determinar en qué medida se relacionan la capacidad de codificación y decodificación facial de emociones y el rendimiento académico en jóvenes universitarios de ingeniería. Para ello, se tomó una muestra probabilística y se midió la capacidad de codificar y decodificar las emociones. Los puntajes obtenidos se relacionaron con los promedios ponderados obtenidos. Lo que se encontró es que la capacidad de codificación y decodificación facial de emociones y el rendimiento académico tienen una correlación poco significativa, negativa o positiva, en jóvenes universitarios de ingeniería. El tipo de relación depende de otra variable "información y práctica sobre comunicación no verbal".
\end{abstract}

Palabras claves: emoción, codificación de emociones, decodificación de emociones, inteligencia no verbal.

\begin{abstract}
This research aimed to determine to what extent the ability of encoding and decoding facial emotions and academic performance in university students is related engineering. To do this, a probability sample was taken and the ability to encode and decode emotions was measured. The scores were related to the weighted averages obtained. What we found is that the ability of encoding and decoding facial emotions and academic performance are not significant, negative or positive correlation engineering in university students. The type of relationship depends on other "and practical information on nonverbal communication" variable.
\end{abstract}

Keywords: emotion, emotions encoding, decoding emotions, nonverbal intelligence.

\section{Introducción}

Desde antes de la modernidad occidental, se ha creído que la inteligencia era solo racional. Bajo esta creencia, las instituciones educativas fueron creadas precisamente para desarrollar la capacidad intelectual de los individuos. Y lo que ellas hicieron cada vez mejor fue elevar y medir los niveles del rendimiento académico de los educandos.

La universidad, si bien su origen se remonta al siglo XII, ha sido una de esas instituciones creadas para el mejoramiento del rendimiento académico de la sociedad. Así, las universidades se convirtieron en los centros por excelencia del pensamiento y actitud modernos. En ellas siempre ha predominado la razón sobre la emoción o la intuición.

La Universidad Nacional Agraria La Molina (UNALM) no ha sido ajena a esto. Por el contrario, puede decirse que en ella se muestra con mayor énfasis, ya que se trata de un centro de estudios tecnológicos que ofrece carreras como Ingeniería Ambiental, Biología, Agronomía, Ingeniería Forestal, Ingeniería Agrícola, Industrias Alimentarias, Economía, Estadística e Informática, Ingeniería en Gestión Empresarial, Pesquería y Zootecnia. El perfil del estudiante molinero promedio es el de una persona inclinada a las ciencias matemáticas y ciencias naturales, mas no a las ciencias humanas ni a las artes. Así, un estudiante de la UNALM es muy competente para resolver problemas matemáticos o aplicar fórmulas, pero poco para la comunicación no verbal; es decir, un estudiante de la UNALM desarrolla lo intelectual, lo racional.

Sin embargo, las teorías de las inteligencias múltiples aparecidas en la segunda mitad del siglo XX han puesto en evidencia la existencia no de una sino de varias inteligencias, entre las que se encuentran la inteligencia emocional, social, no verbal y comunicativa. Éstas consisten, en general, en la capacidad de controlar y comunicar las emociones a fin de establecer relaciones eficaces y eficientes con los demás. A esto debe añadirse el hecho que el mundo del siglo XXI exige cada vez más la necesidad de estos tipos de inteligencias. La inteligencia lingüística y la inteligencia lógico-matemática no son suficientes en la sociedad del siglo XXI; se requiere también de las otras, sobre todo de la emocional, social, no verbal y comunicativa. 
Entonces, las universidades, que forman personas y no máquinas, deben tomar en cuenta los distintos tipos de inteligencias y formar profesionales que han de desenvolverse en la sociedad, relacionándose eficientemente con muchos otros. Sin duda, la responsabilidad de desarrollar las otras inteligencias también alcanza a universidades como La Molina.

Pues bien, la inteligencia emocional, social, no verbal y comunicativa tiene que ver con las emociones y el lenguaje del cuerpo. Pero estos dos elementos son bastante amplios, por lo que es necesario reducir con fines de estudio.

Los seres humanos comunicamos las emociones con palabras, pero también con signos no verbales. De hecho, cuando comunicamos las emociones con palabras, es el paralenguaje (características de la voz) el que comunica más. Pero más que ambos, es el rostro humano el que expresa y comunica las emociones. Los movimientos faciales son los que expresan y comunican las emociones básicas y universales como la alegría, la tristeza, la ira, la sorpresa, el miedo y el asco. Por eso, el objeto de estudio de esta investigación ha sido la capacidad de codificación y decodificación de esas emociones a través del rostro y su relación con el rendimiento académico.

En tal sentido, se planteó el siguiente problema general de investigación: ¿En qué medida se relacionan la capacidad de codificación y decodificación facial de emociones (alegría, tristeza, sorpresa, asco, ira y miedo) y el rendimiento académico en jóvenes universitarios de ingeniería? El objetivo fue: Determinar en qué medida se relacionan la capacidad de codificación y decodificación facial de emociones (alegría, tristeza, sorpresa, asco, ira y miedo) y el rendimiento académico en jóvenes universitarios de ingeniería. Y se partió de la hipótesis: La capacidad de codificación y decodificación facial de emociones (alegría, tristeza, sorpresa, asco, ira y miedo) no se relaciona con el rendimiento académico en jóvenes universitarios de ingeniería.

\section{Metodología}

\subsection{Lugar de investigación}

La investigación se realizó en el campus de la Universidad Nacional Agraria La Molina, en el distrito La Molina, provincia de Lima.

\subsection{Diseño de la investigación}

Para responder las preguntas formuladas en el planteamiento del problema de investigación, se ha elegido el diseño experimental, ya que una de las variables ha sido controlada "capacidad de codificación de emociones expresadas en el rostro".
Este diseño experimental ha sido con un enfoque mixto, pero con predominio de lo cuantitativo en la recolección e interpretación de los datos. Afirmamos que ha sido mixto porque también se ha recogido y valorado datos de modo cualitativo.

La expresión de emociones, como un conjunto de signos que expresan las emociones que experimenta el sujeto, se aprehende mejor con un enfoque cualitativo. El triángulo (significante - significado - referente) propuesto por los semiólogos clásicos, desde Charles Sanders Peirce y Ferdinad de Sassure hasta Greimas, es pertinente.

Sin embargo, para estimar la capacidad de codificación es necesario un enfoque cuantitativo.

\subsection{Fases del diseño de la investigación}

Primera fase: En esta primera fase se relacionó la variable "Capacidad de decodificación de emociones expresadas en el rostro" con la variable "Rendimiento académico". No se relacionó la variable "Capacidad de codificación facial de emociones" con la variable "Rendimiento académico", porque se careció de financiamiento y porque el autor ya lo hizo en una investigación anterior.

Segunda fase: En esta segunda fase se volvió a relacionar la variable "capacidad de decodificación de emociones expresadas en el rostro" con la variable "Rendimiento académico", pero después de ofrecerle a los participantes un breve curso de 10 horas sobre la comunicación no verbal (en este curso se incluyó el tema de la expresión de las emociones, empero no fue el único). También, se relacionó la variable "capacidad de codificación facial de emociones" con la variable "Rendimiento académico.

\subsection{Población y muestra}

La población ha estado conformada por los alumnos universitarios del II Ciclo 2013-II de la Universidad Nacional Agraria La Molina que sumaron en total 480.

Se usó un muestreo probabilístico proporcional, resultado de la aplicación de una fórmula estadística. De este modo, se tomó como muestra a 77 estudiantes. De los cuales, 38 fueron mujeres y 39 varones, cuyas edades fluctuaron entre 18 y 22 años.

\subsection{Instrumentos de colecta de datos}

Para la primera parte del trabajo se aplicó un test propuesto por Paul Ekman en su libro EI rostro de las emociones. Signos que revelan significado más allá de las palabras. A este test se le asignó un puntaje de 0 a 20 para poder compararlo con el promedio ponderado del rendimiento académico.

Y para segunda parte de esta investigación, se solicitó a cada participante que expresara cada una de las seis emociones universales y se tomaran una fotografía. Las fotografías a evaluar tuvieron las siguientes características: 
emoción expresada solamente con movimientos faciales, plano busto de frente, en colores, fondo blanco, de $6 \mathrm{~cm}$. de ancho por $6 \mathrm{~cm}$. de largo. Las fotografías se pusieron en un fólder para que fueran evaluadas por un grupo de 50 personas, tanto hombres como mujeres. Asimismo, esas fotografías fueron evaluadas por el investigador ponderando los movimientos de cada zona del rostro.

Para la evaluación de las expresiones faciales de cada zona del rostro se usó la escala de Likert, asignando un puntaje de 1 a 5 .

\subsection{Procedimiento de análisis de datos}

Los datos se han procesado usando técnicas estadísticas en una hoja de cálculo de Excel. Se han calculado las frecuencias relativas y absolutas, los porcentajes respectivos y la media. Asimismo, se ha hallado el coeficiente de correlación entre las variables para probar las hipótesis.

\section{Resultados y discusión}

Los resultados encontrados en esta investigación y la interpretación respectiva los presentaremos en cinco partes. Primero, sobre el nivel de capacidad de decodificación y codificación. Segundo, lo referido a la decodificación y su relación con el rendimiento académico. Tercero, respecto a la codificación y el rendimiento académico. Cuarto, la respuesta a por qué estos resultados. Y quinto, para qué estos resultados.

\subsection{Nivel de decodificación y codificación}

Los porcentajes de aciertos en la decodificación, antes y después de las lecciones de comunicación no verbal, revelan el nivel de los estudiantes en cuanto a lectura e interpretación de las emociones expresadas en el rostro. Como se observa en la Tabla 1, el nivel de los alumnos de ingeniería es medio.

Si se analizan los resultados por emoción, se observa que el desempeño en la decodificación de la alegría es muy alto y es esta cifra la que eleva el resultado final. Si se descuentan las emociones consideradas fáciles de reconocer (alegría y sorpresa ${ }^{2}$ ), el nivel resulta bajo, mucho más bajo que el promedio del rendimiento académico de 12.3 puntos.

Tabla 1. Nivel de decodificación de emociones antes y después de lecciones de comunicación no verbal.

\begin{tabular}{ccccc}
\hline Emoción & $\begin{array}{c}\% \text { de } \\
\text { aciertos } \\
\text { antes de } \\
\text { lecciones }\end{array}$ & $\begin{array}{c}\text { Nivel antes de } \\
\text { de lecciones después de } \\
\text { aciertos } \\
\text { lecciones }\end{array}$ & $\begin{array}{c}\text { Nivel } \\
\text { después de } \\
\text { lecciones }\end{array}$ \\
\hline Alegría & 92.2 & muy alto & 94.5 & muy alto \\
Sorpresa & 87.4 & muy alto & 88.6 & muy alto \\
\hline \hline
\end{tabular}

${ }^{2}$ En una investigación realizada por el autor de ésta, se comprobó que la alegría y la sorpresa son emociones fáciles de expresar y reconocer, mientras que la tristeza, el asco, la ira y el miedo son emociones difíciles (Castillo, 2012).
Continuacion de tabla 1

\begin{tabular}{ccccc}
\hline Tristeza & 62.99 & alto & 64.65 & alto \\
Asco & 43.1 & medio & 47.3 & medio \\
Ira & 19.74 & muy bajo & 22.6 & bajo \\
Miedo & 33.12 & bajo & 40.9 & medio \\
\hline Media & $\mathbf{5 6 . 4 3}$ & medio & $\mathbf{5 9 . 7 6}$ & medio \\
\hline
\end{tabular}

Fuente: elaboración propia.

La Tabla 1 también muestra que hubo un incremento, aunque poco significativo, de los aciertos después de haber recibido el curso de comunicación no verbal (intervención de la variable "información y práctica sobre comunicación no verbal"). Si traducimos a una escala de 0 a 20, que es la que se utiliza en la calificación de los estudiantes en la UNALM, se podrá notar que se pasó de una mediana de 11.3 puntos a una de 11.9 puntos. En ambos casos inferior al promedio ponderado del rendimiento académico de 12.3 puntos de los participantes.

El Gráfico 1 muestra el incremento de los porcentajes de aciertos de los alumnos al momento de reconocer las emociones básicas después de la intervención de la variable "información y práctica sobre comunicación no verbal". Como se observa, las barras rojas, que representan los porcentajes de aciertos después de las lecciones de comunicación no verbal, son ligeramente más altas que las azules. Lo que está revelando este ascenso es que en la escuela o la universidad se mejora la capacidad de decodificación no verbal.

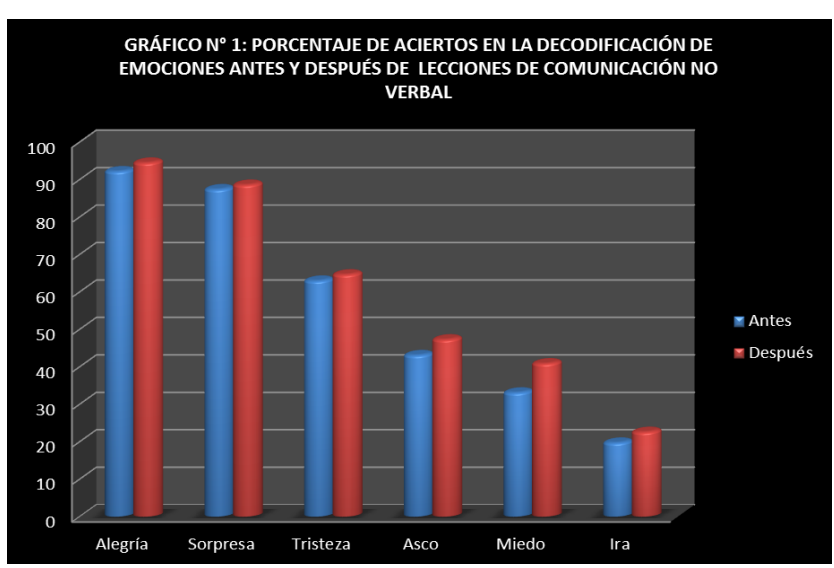

Figura 1. Porcentaje de aciertos en la docodificacion de emociones antes y después de lecciones de comunicación no verbal.

Fuente: Elaboración propia

En cuanto a la codificación facial de emociones los resultados fueron los siguientes. La mediana de los puntajes obtenidos por los participantes en la codificación de las emociones fue 10.2. Esta mediana, obtenida después de recibir lecciones de expresión de emociones, es inferior al 12.3 de promedio ponderado de rendimiento académico (ver Tabla 2). Esto muestra que los estudiantes de ingeniería tienen un desempeño desaprobatorio en la codificación facial de emociones. 
Tabla 2. Mediana de los puntajes de codificación de emociones por zona del rostro (Escala de 0 a 20).

\begin{tabular}{lcccc}
\hline $\begin{array}{c}\text { Emoción/ } \\
\text { zona }\end{array}$ & Superior & Intermedia & Inferior & Total \\
\hline Alegría & 12.64 & 12.76 & 13.16 & 13 \\
Tristeza & 9.28 & 9.76 & 10.6 & 10 \\
Asco & 9.16 & 9.6 & 10.84 & 10 \\
Miedo & 8.2 & 8.4 & 8.32 & 8 \\
Sorpresa & 10.32 & 10.12 & 10.24 & 10 \\
Ira & 10.24 & 10 & 10.44 & 10 \\
Total & $\mathbf{1 0}$ & $\mathbf{1 0}$ & $\mathbf{1 1}$ & $\mathbf{1 0}$ \\
\hline
\end{tabular}

Fuente: Elaboración propia.

Si el análisis se hace por emociones, se podrá comprobar que solamente en la codificación de la alegría se logra una nota aprobatoria (13). En las demás emociones el puntaje es desaprobatorio, entre 8 y 10 (ver Tabla 2).

Todo esto revelaría que los estudiantes de ingeniería tienen un nivel bajo en la codificación de las emociones. Entonces, tanto en la decodificación como en la codificación de emociones básicas, el desempeño de los alumnos es mucho menor que su desempeño en las áreas matemáticas, verbales y lógicas.

La Tabla 2 revela también aspectos específicos importantes del desempeño de los estudiantes de ingeniería en la codificación de emociones básicas.

Asumimos que el rostro tiene zonas donde se expresan las emociones y cada zona expresa más una emoción que otra. A su vez, unas zonas son más fáciles o difíciles de gesticularlas. Estas zonas son: superior (frente y cejas), intermedia (ojos) e inferior (nariz y boca).

Para los estudiantes de ingeniería, fue más fácil codificar las emociones con los movimientos estereotipados que probablemente hayan visto frecuentemente en los medios visuales de comunicación. Los promedios más altos en determinadas zonas del rostro así lo confirman. La alegría lo expresan principalmente con las comisuras de los labios hacia atrás y hacia arriba. La tristeza, con las comisuras de los labios hacia abajo. El asco, con la arruga de la nariz. La sorpresa, con las cejas arqueadas y la boca abierta. Y la ira, con las cejas contraídas y los labios apretados y rectos. El miedo no tiene un movimiento facial característico reconocido por los estudiantes, pero ellos tienden a codificarlo más con los ojos abiertos y la boca también abierta.

\subsection{Decodificación y rendimiento académico}

En este aspecto, se partió de la hipótesis general "la capacidad de decodificación facial de emociones no se relaciona con el rendimiento académico en alumnos de ingeniería". Sin embargo, lo hallado (los diagramas de dispersión y los coeficientes de correlación de -0.17 y
0.16) permite sostener que sí existe una relación entre estas dos variables, aunque, claro, poco significativa (ver Tabla 3). Entonces, la hipótesis general ha sido rechazada. La relación entre la capacidad de decodificación facial de emociones y el rendimiento académico en los alumnos de ingeniería tiene una característica importante: puede ser negativa o positiva. La variable "información y práctica sobre comunicación no verbal" determina el tipo de relación ${ }^{3}$.

Cuando la persona no ha tenido información respecto de la comunicación no verbal, existe una ligera tendencia a que haya una relación inversa entre la capacidad de decodificar las emociones expresadas en el rostro de otra persona y el rendimiento académico. Es decir, a mayor rendimiento académico, menor capacidad de leer las emociones de los otros. Es cierto que no se trata de una marcada correlación o de una correlación significativa, por eso se afirma ligera tendencia (ver Tabla 3).

Cuando la persona ha tenido información respecto de la comunicación no verbal, se presenta una ligera relación positiva entre la capacidad de decodificar las emociones expresadas en el rostro y el rendimiento académico. En otras palabras, los más estudiosos tienden a mejorar sus niveles de lectura de las emociones. Las personas racionalizan su percepción: cuanto más saben sobre la expresión facial de emociones, mejor las reconocen en los demás (ver Tabla 3). Claro, otra vez, se trata de una correlación poco significativa.

Aunque poco significativa, lo que llama la atención es la variación de -0.17 a 0.16 , de negativo a positivo, como consecuencia de la intervención de la variable "información y práctica sobre comunicación no verbal".

Tabla 3. Correlación entre la capacidad de decodificar las emociones y el rendimiento académico.

\begin{tabular}{lc}
\hline & $\begin{array}{c}\text { Coeficiente de } \\
\text { correlación }\end{array}$ \\
\hline Antes de recibir información & -0.17 \\
Después de recibir información & 0.16 \\
\hline Fuente:
\end{tabular}

Cuando el análisis se hace por sexo, la correlación pasa de inversa a proporcional claramente en ambos casos, aunque en el caso de los varones es más significativo el cambio. Esto merecería otro estudio y nuevas pruebas, ya que el consenso de los científicos es que las mujeres son más competentes en la decodificación que los varones (ver Tabla 4). El hecho que en el caso femenino la variación sea menor significa que las mujeres con menor rendimiento académico también tienen un buen desempeño en la comunicación no verbal.

\footnotetext{
${ }^{3}$ Como ya se explicó en la metodología, para esta investigación se realizó un curso breve de 10 horas sobre comunicación no verbal. Se explicaron los aspectos generales y uno de los temas tratados, pero no el exclusivo, fue la interpretación y expresión facial de las emociones básicas.
} 
Tabla 4. Correlación entre la capacidad de decodificar las emociones y el rendimiento académico.

\begin{tabular}{lccc}
\hline & & $\begin{array}{c}\text { Coeficiente } \\
\text { de correlación } \\
\text { (mujeres) }\end{array}$ & $\begin{array}{c}\text { Coeficiente } \\
\text { de correlación } \\
\text { (varones) }\end{array}$ \\
\hline $\begin{array}{l}\text { Antes de recibir } \\
\text { información }\end{array}$ & -0.13 & -0.2 \\
$\begin{array}{l}\text { Después de } \\
\text { información }\end{array}$ & 0.14 & 0.17 \\
\hline
\end{tabular}

Fuente: elaboración propia.

Al iniciar esta investigación, se plantearon las hipótesis específicas que se resumen así: la capacidad de decodificación facial de la alegría, tristeza, asco, miedo, sorpresa e ira no se relaciona con el rendimiento académico en jóvenes universitarios de ingeniería. No obstante, los coeficientes de correlación hallados para cada una de las emociones básicas (ver Tabla 5) nos llevan a rechazar estas hipótesis y afirmar que sí tienen relación. Si bien se trata de correlaciones muy débiles, es importante notar el cambio de negativo a positivo en todas las emociones y que de alguna manera respaldan la correlación general hallada.

Tabla 5. Coeficientes de correlación entre la capacidad de decodificar las emociones y el rendimiento académico antes y después de las lecciones de comunicación no verbal.

\begin{tabular}{lcc}
\hline & Antes & Después \\
\hline Alegría & -0.11 & 0.05 \\
Sorpresa & -0.04 & 0.05 \\
Tristeza & -0.03 & 0.04 \\
Asco & -0.05 & 0.16 \\
Ira & -0.12 & 0.22 \\
Miedo & -0.01 & 0.14 \\
\hline
\end{tabular}

Fuente: elaboración propia.

Lo que se concluye de estos primeros resultados es que sí existe una correlación, aunque poco significativa, entre la capacidad de decodificar las emociones y el rendimiento académico. No se puede afirmar, entonces, que no tienen ninguna relación.

Otra afirmación que puede hacerse de esta parte es que se aprende en la escuela a reconocer las emociones que se expresan en el rostro. El estudio y la práctica mejora la competencia para decodificar las emociones. Probablemente no se alcance un nivel óptimo, como ocurre en lo intelectual, pero es claro el incremento del desempeño. Estas conclusiones confirman las afirmaciones recogidas por Mark Knapp.

“(...) parece ser mínima la relación existente entre inteligencia y otras medidas verbales y la capacidad de decodificación de señales no verbales (...) los actores, los estudiantes de conductas no verbales y los estudiantes de artes visuales tienden a puntuar mejor en los tests de capacidad de decodificación no verbal, pero de aquellos que reciban dentro de su grupo profesional la calificación de excelente se puede esperar que posean buenas facultades para la decodificación no verbal" (Knapp, 1985: 336).

Lo anterior se refuerza observando la evolución del nivel de decodificación de las emociones antes y después de recibir información acerca de la comunicación no verbal. El nivel de decodificación de emociones antes de recibir las lecciones de comunicación no verbal es relativamente menor que el nivel de decodificación después de recibir lecciones de comunicación no verbal (ver Tabla 1). Ciertamente, como se ve en la Tabla 1, no se trata de una mejora significativa, pero creemos que se debe al tiempo de estudio y práctica. Para que haya una mejora significativa son necesarias muchas horas. Lo que resultó notorio cuando se procesaron los datos es que las personas con mayor rendimiento académico mejoraron más.

\subsection{Codificación y rendimiento académico}

Lo que se ha afirmado frecuentemente es que la expresión facial de emociones no se relaciona con el rendimiento académico. Por eso, en esta investigación se partió de la hipótesis general "la capacidad de codificación facial de emociones no se relaciona con el rendimiento académico en jóvenes universitarios de ingeniería".

Sin embargo, a la luz del diagrama de dispersión y del coeficiente de correlación positivo (0.074) obtenidos en esta investigación y lo que se encontró en cuanto a la capacidad de decodificación, se puede afirmar categóricamente que sí existe una relación entre el rendimiento académico y la capacidad de codificar emociones, aunque débil y poco significativa.

Esta relación varía con la intervención de otras variables. En este trabajo realizamos solo una prueba después de incluir la variable "información y práctica sobre comunicación no verbal" y los resultados fueron los siguientes:

Cuando se añade la variable interviniente "información y práctica sobre comunicación no verbal", al parecer sí tienen ligeramente una relación directa aunque poco significativa. Es decir, a más rendimiento académico más capacidad para codificar.

En esta investigación se obtuvo un coeficiente de 0.074 , empero se asume que se debe al escaso tiempo de preparación, lo que lleva a pensar que ese coeficiente positivo puede incrementarse. Lo notorio en el procesamiento de datos ha sido que los que tenían 
mejores notas tendían a esforzarse más en la expresión de emociones y lo hacían mejor, aunque a veces se equivocaban por buscar el movimiento adecuado.

Lo que se debe precisar es que a diferencia de lo que ocurre con la decodificación, la codificación requiere mayor tiempo de práctica y mayores detalles en las lecciones. Un curso completo de comunicación no verbal sería lo ideal, porque no es fácil aprender a mover los músculos de la cara encargados de la expresión de las emociones, sin reconocerlos previamente y sin mucha práctica.

Tabla 6. Correlación entre la capacidad de codificar las emociones y el rendimiento académico.

\begin{tabular}{ccc}
\hline & $\begin{array}{c}\text { Coeficiente de } \\
\text { correlación (mujeres) }\end{array}$ & $\begin{array}{c}\text { Coeficiente } \\
\text { de correlación } \\
\text { (varones) }\end{array}$ \\
\hline $\begin{array}{c}\text { Después } \\
\text { de recibir } \\
\text { información }\end{array}$ & 0.041 & 0.123 \\
\hline Fuente: Elaboración propia &
\end{tabular}

Al observar los resultados específicos por cada emoción, existen variaciones que son importantes describirlas y explicarlas. Se partió de la hipótesis de que la capacidad de codificar la alegría, la sorpresa, la ira, la tristeza, el miedo y el asco no tenía relación con el rendimiento académico. Sin embargo, los resultados hallados muestran que sí tienen relación. Esto refuerza la conclusión general obtenida en este aspecto.

En el caso de la alegría, lo que se obtuvo es un coeficiente de correlación de -0.1. Es decir, inversa. En el caso de la sorpresa, se obtuvo un coeficiente 0.0 , lo cual indica que no existe relación. En cambio, en los casos de la tristeza, el miedo, la ira y el asco, los coeficientes fueron positivos, es decir que existen relaciones débilmente directas (ver Tabla 7).

Tabla 7. Correlación entre competencia emocional y académica.

\begin{tabular}{cc}
\hline Emoción & Coef. de correl. \\
\hline Alegría & -0.1 \\
Tristeza & 0.1 \\
Asco & 0.1 \\
Miedo & 0.09 \\
Sorpresa & 0 \\
Ira & 0.07 \\
\hline
\end{tabular}

Fuente: Elaboración propia

Estos resultados se explican de la siguiente manera.

En otras investigaciones y en una anterior realizada por el autor de ésta, se encontraron que por la facilidad o dificultad para expresar emociones, las emociones básicas se pueden dividir en dos: las difíciles y las fáciles. Entre las fáciles está la alegría y, en menor medida, la sorpresa. Entre las difíciles, se encuentran el miedo y, en menor medida, la ira, la tristeza y el asco.

Entonces, antes y después de una lección de comunicación no verbal, la alegría suele ser fácil de expresar. Por lo tanto, el coeficiente de correlación de -0.1 prueba que los que tienen bajos promedios académicos también pueden tener un buen desempeño en la expresión no verbal, ya que la mayoría sabe expresar la alegría, porque tiene claro cuáles son los movimientos faciales que expresan esta emoción y son conscientes de ello ${ }^{4}$. Sin embargo, el coeficiente de correlación de -0.1 también prueba que los que tienen alto rendimiento académico tienen menos capacidad para expresar la alegría, pero esto al parecer es consecuencia del hecho de que los alumnos con alto rendimiento académico buscan expresarlo mejor y se equivocan en el intento. Ellos distinguen los distintos tipos de sonrisas y reconocen las auténticas, pero para expresarlas requieren mayor preparación (ver Tabla 7).

Cuando se trata de las emociones difíciles, el coeficiente de correlación es positivo. Es decir, aunque débilmente, a mayor promedio académico mayor promedio en la expresión de emociones difíciles. Los más estudiosos tienen mejor desempeño. Esto revela que, aunque con muchas dificultades, los alumnos que tienen alto promedio académico expresan mejor el miedo, la ira, la tristeza y el asco, consideradas emociones difíciles (ver Tabla 7).

\section{4 ¿Por qué estos resultados?}

Los resultados obtenidos plantean las siguientes preguntas: ¿Por qué la correlación es muy débil y poco significativa? ¿Por qué la mejora es poco significativa cuando interviene la variable "información y práctica sobre comunicación no verbal"? Postulamos que existen cuatro razones, estrechamente relacionadas, que responden a estas preguntas: el perfil del codificador y decodificador no verbal, los límites de los tipos de inteligencia, los efectos de una educación centrada en lo verbal que termina inhibiendo las capacidades no verbales y el tiempo de estudio y preparación. A continuación, explicaremos cada uno de estos factores.

\subsubsection{El perfil de codificador y decodificador no verbal}

La capacidad de codificar y decodificar las emociones expresadas en el rostro dependen también de muchas otras variables, tales como el sexo, la edad, la autonomía,

\footnotetext{
${ }^{4}$ En otro trabajo de investigación hemos explicado esto afirmando: "E hecho de que la alegría sea la emoción más fácil de expresar, fingir y reconocer, y que el miedo sea la más difícil, revela claramente la influencia cultural: en Occidente la alegría se valora más, ya que favorece los vínculos interpersonales. Por eso, se aprende a expresarla, reconocerla, fingirla o disimularla desde muy niños. En cambio, el miedo es escondido, disfrazado, rechazado y sancionado. Por ejemplo, a los varones se les prohíbe tener miedo" (Castillo, 2012).
} 
la extroversión o introversión de los individuos. No se relacionan únicamente con el rendimiento académico.

Mark Knapp reseñó las conclusiones de diferentes investigadores respecto a los buenos decodificadores:

“1) como grupo, las mujeres tienden a ser mejores decodificadoras que los varones; 2) las capacidades decodificadoras tienden a aumentar con la edad hasta los veintitantos años; 3) parece ser mínima la relación existente entre inteligencia y otras medidas verbales y la capacidad de decodificación de señales no verbales; 4) la personalidad de los decodificadores idóneos parece reflejar extroversión, sociabilidad, autocontrol y juicios de eficacia interpersonal por parte de los demás; 5) los actores, los estudiantes de conductas no verbales y los estudiantes de artes visuales tienden a puntuar mejor en los tests de capacidad de decodificación no verbal, pero de aquellos que reciban dentro de su grupo profesional la calificación de excelente se puede esperar que posean buenas facultades para la decodificación no verbal; 6) los tests en los que se utilizan estímulos faciales, corporales y vocales típicos de sujetos estadounidenses provocan los mayores puntajes en las culturas más similares a la norteamericana, pero las calificaciones de acierto sugieren la posibilidad de un componente multicultural en la conducta de decodificación no verbal; y 7) la excitación fisiológica y la práctica también parecen incrementar la capacidad de decodificación de una persona" (Knapp, 1985: 336).

Asimismo, Mark Knapp afirma sobre los codificadores competentes:

“(...) las personas que ejercen un gran 'control de sí mismas' están en mejores condiciones para emitir información emocional a través de los canales vocales y faciales. Los 'introvertidos' constituyen estímulos más pobres para los demás que los 'extrovertidos'. Denominaremos introvertidos a aquellas personas que tienden a reprimir sus reacciones emocionales, a 'guardarse sus sentimientos' (...) los niños que resultaron eficaces emisores eran abiertos, comunicativos, activos, sociables y algo autoritarios e impulsivos. Los emisores ineficaces mostraban tendencia a jugar solos, eran cerrados, pasivos, tímidos, contenidos y se consideró que poseían más espíritu de cooperación” (Knapp, 1985: 333).

Entonces, estas reseñas que recogemos de Mark Knapp, en la que se observa que la capacidad para la comunicación no verbal se relaciona con muchas otras capacidades o características personales, explicaría por qué la correlación es muy débil y poco significativa y por qué la mejora es poco significativa cuando interviene la variable "información y práctica sobre comunicación no verbal". En esta investigación solamente se ha relacionado la capacidad de codificar y decodificar las emociones expresadas en el rostro con el rendimiento académico, una de las tantas variables.

\subsubsection{Los límites de los tipos de inteligencias}

La población estudiada estuvo conformada por estudiantes de ingenierías que tienen un perfil que no necesariamente se caracteriza por el óptimo desempeño en la comunicación tanto verbal como no verbal. Se trata de jóvenes que han sido seleccionados a través de un examen de sus habilidades matemáticas y verbales ${ }^{5}$, dadas las particularidades de las carreras, pero no con un examen de inteligencia emocional ni de inteligencia no verbal. Así, estos jóvenes destacan en ciencias naturales y matemáticas, mas no siempre descuellan en la comunicación. Esto se ha podido comprobar con el nivel de codificación y decodificación presentado en el acápite 4.1.

Si bien en esta investigación se encontró que estudiando y practicando se puede mejorar la capacidad de decodificación y la codificación de emociones y en eso la universidad puede aportar mucho, también es cierto que existen otras variables de las cuales depende esa capacidad.

Uno de los límites que encontrará el desarrollo de la competencia no verbal es el tipo de inteligencia que poseen las personas y el hecho de que las personas no se pueden desempeñar con el mismo nivel de competencia en todas las áreas. Asumimos que las personas tienen más capacidad para desarrollarse en unas áreas que en otras.

"Aunque no dudo que algunas personas puedan tener el potencial necesario para destacar en más de un ámbito, me opongo resueltamente a la idea de las grandes facultades generales. En mi opinión, la mente tiene la capacidad de tratar distintos tipos de contenidos, pero resulta en extremo improbable que la capacidad de una persona para abordar un contenido permita predecir su facilidad en otros campos. En otras palabras, es de esperar que el genio (y afortiori, el desempeño cotidiano) se incline hacia contenidos particulares: los seres humanos han evolucionado para mostrar distintas inteligencias y no para recurrir de diversas maneras a una sola inteligencia flexible" (Gardner, 1995: 5).

Entonces, la escuela o la universidad pueden desarrollar la competencia no verbal de los alumnos pero hasta cierto nivel, más no sería posible. Una persona con inteligencia lingüística o lógico-matemática probablemente mejore su comunicación y reconocimiento de emociones, pero hasta cierto nivel. No creemos que se pueda pasar de un coeficiente de correlación negativa perfecta de -1 a un

${ }^{5}$ Para ingresar a la UNALM se toma un examen de admisión a los postulantes. En general, compiten 6 alumnos por una vacante. En carreras como Ingeniería Ambiental, la competencia puede ser de 81 a 1. Los ingresantes tienen un nivel alto de desempeño en las áreas de ciencias naturales y matemáticas. 
coeficiente de correlación positivo perfecto de +1 por mucho tiempo de preparación que haya tenido, a menos que en su perfil se encuentren todas las características del buen codificador o decodificador no verbal antes mencionados.

Sin embargo, sostenemos que la universidad puede desarrollar la competencia comunicativa no verbal de sus alumnos lo suficiente como para que estos mejoren sus interrelaciones interpersonales en el mundo laboral y profesional. Para esto un curso completo de CNV es necesario.

\subsubsection{Los efectos de una educación solamente verbal}

En las escuelas y las universidades, la comunicación no verbal no es un tema central; es el lenguaje verbal el que se estudia más.

"Nuestra formación en la escuela, en la universidad, se ha articulado alrededor del lenguaje verbal escrito. Nadie nos ha enseñado la gramática del lenguaje corporal o los recursos vocales que podemos utilizar. En la educación primaria y secundaria se ha priorizado el análisis del lenguaje verbal y su estudio teórico en lugar de fomentar la oralidad, con la intervención correspondiente de la voz y el lenguaje corporal. Nos han mostrado sólo una parte de la realidad" (Baró, 2012: 23).

Erróneamente creemos que lo más importante en la comunicación es lo que decimos con palabras.

"Con nuestra educación predominantemente racional creemos que lo más importante es el mensaje verbal. Incluso tenemos la percepción de que es el único mensaje que enviamos, tal es la atención que ponemos en él: conceptos, datos, argumentos, opiniones, emociones, descritas En cambio, ignoramos los mensajes que envuelven a los anteriores y que inevitablemente transmitimos: ilusión, compromiso, desánimo, nerviosismo, confianza (...)" (Baró, 2012: 23-24).

Eduardo Punset, destacado divulgador científico, ha sido más categórico al describir la realidad de la educación contemporánea en este aspecto.

"Entre las múltiples lecciones que estamos llamados a desaprender para adaptarnos a los tiempos que nos está tocando vivir, una de las más importantes tiene que ver con el desorbitado valor que en el pasado le dimos a la comunicación verbal, desequilibrio que continúa presente aún en nuestros días" (Punset, 2012: 152).

Esto, sin duda, no implica restarle importancia a lo verbal, que ha sido reconocido como fundamental para la evolución humana, al punto que sin palabras el homo sapiens no se hubiera distanciado del resto de homínidos.

"Sin embargo, con ser crucial el factor verbal para hacer realidad al hombre tal y como hoy lo conocemos, la enorme atención prestada por nuestra cultura hacia la irrupción del lenguaje y sus consecuencias ha tenido un reverso en forma de déficit: hemos descuidado la comunicación que somos capaces de entablar sin necesidad de acudir a las palabras. Sólo ahora, cuando la neurociencia ha entrado con lupa de aumento en el interior del cerebro y de la psique, empezamos a admitir que hemos puesto demasiados huevos en el cesto del lenguaje oral y muy pocos en el de la comunicación no verbal" (Punset, 2012: 152).

Pues, bien, la educación orientada más a desarrollar la inteligencia lingüística y lógico matemática, termina parcialmente inhibiendo las capacidades naturales para la expresión y reconocimiento de emociones con las que todos nacemos. Claro, no se nace con la capacidad emocional desarrollada al máximo, pero se tiene una base que a lo largo de los años, hasta la etapa de la juventud, se va perfeccionando. Cuando a un niño de cinco años se le pide que exprese y reconozca las emociones básicas y universales lo hace con cierta destreza. Al parecer es este desarrollo el que se altera con la educación tradicional basada en lo verbal escrito. Alan García Pérez, quien no ha teorizado ni investigado sobre la comunicación no verbal, pero que ha demostrado ser muy competente, explica esto desde su propia experiencia.

"(...) todos sabemos comunicar al comienzo. Un niño llora, sonríe, se expresa, pero a partir de ese momento la comunidad de expresión se divide y algunos sabrán expresar y otros no, porque la formación familiar, el orden social y la educación conducen a inhibir la expresión. Todos los niños mueven los ojos, las manos, y esos son los movimientos "mágicos" de los que habla la psicología. Todos sus juegos están concentrados en movimientos corporales; es decir, se expresan. Pero luego son limitados por órdenes sociales, como "no llorar", "no interrumpir" a los mayores", "escuchar en silencio mientras el maestro habla durante una hora". Es la cultura del silencio, la declamación con las manos pegadas al cuerpo y el uso del telepromter

Así, el niño que aprendió el ritmo de su propio llanto, que era para él un trabajo productivo por el que obtuvo gratificación, el niño que recogió la melodía de la canción de cuna o la cadencia del Padre Nuestro, debe ser, después de inhibido, reentrenado en esas aptitudes por un adiestramiento impuesto y mecánico. Bien sabemos que un infante recién nacido tiene la capacidad de nadar bajo el agua, pero en los años posteriores será alejado del peligro y deberá, en la mayoría de los casos forzadamente, reaprender a nadar. Igual ocurre con la 
transmisión emocional, pues se reprime y luego se intenta recuperar tardíamente en el diván del psiquiatra" (García, 2012: 62).

Podemos afirmar, entonces, que revertir los efectos de la educación basada en la palabra escrita, no es fácil ni rápido.

Lo que hemos tenido en esta investigación ha sido un grupo de participantes que han recibido una educación basada en lo verbal escrito y, principalmente, numérico. En los planes de estudio de la UNALM no existen cursos orientados a mejorar la inteligencia comunicativa no verbal ni la inteligencia emocional ni la inteligencia social. Los pocos cursos orientados a mejorar la comunicación son Lengua, Comunicación y Redacción Técnica, todos basados en el estudio y la práctica del idioma español escrito. Y esto pasa no solamente en la UNALM sino que es, como ya lo mencionamos, una característica de la educación superior moderna y de todos los niveles.

En el caso de los alumnos de la UNALM, a la educación primaria y secundaria puramente verbal hay que sumarle un plan de estudio universitario también centrado en lo verbal y lo numérico. Y como consecuencia de todo ello, se da la inhibición de las capacidades no verbales o el escaso desarrollo de éstas.

\subsubsection{El tiempo de estudio y preparación}

La competencia comunicativa no verbal, como ya lo definimos, es la capacidad de emitir mensajes no verbales correctos y la habilidad para interpretar adecuadamente los mensajes no verbales de los demás. Y adquirirla requiere muchas horas de estudio y práctica.

Según Teresa Baró la competencia no verbal se adquiere en cuatro etapas:

$1^{\circ}$ Ignorancia inconsciente: Es la etapa en la que no se sabe que no se sabe. Es decir, no se ha escuchado ni leído acerca de la comunicación no verbal. Hasta que en algún momento te enteras.

$2^{\circ}$ Ignorancia consciente: Ya se sabe que no se sabe acerca de la comunicación no verbal o que se puede aprender.

$3^{\circ}$ Conocimiento consciente: Es la etapa en la que se lee y estudia sobre la comunicación no verbal. Se sabe tanto que se puede dar conferencias sobre esta materia. Es la etapa en la que se estudia y se practica.

$4^{\circ}$ Conocimiento inconsciente: Esta es la etapa final del aprendizaje, en la que ya se ha interiorizado los conocimientos que los movimientos corporales fluyen de manera natural, que inclusive hay que hacer un esfuerzo para explicar en qué consisten.
"Ha llegado a formar parte de tu conocimiento, de tus habilidades inconscientes. Esta capacidad te permite destinar tu energía o centrar tu atención en otras cosas" (Baró, 2012: 291-292).

¿Cuánto tiempo de estudio y práctica requerirá un grupo de jóvenes, que estudian ingeniería y que han recibido a lo largo de sus vidas una educación basada en los números y la palabra, para adquirir la competencia no verbal o desarrollar la que tienen? Sin duda, no 10 horas, sino mucho más.

Lo que ha sucedido durante esta investigación es que no se ha ofrecido a los participantes un curso completo de codificación y decodificación facial de emociones, sino simplemente conocimientos generales. Los estudiantes se quedaron apenas iniciando la tercera etapa. Esto explica por qué lo cambios fueron poco significativos. Si se busca una correlación positiva media, que es lo que suponemos que se puede lograr, es necesario un curso integral de comunicación no verbal.

\section{Conclusiones}

La capacidad de codificación y decodificación facial de emociones y el rendimiento académico tienen una correlación poco significativa negativa o positiva en jóvenes universitarios de ingeniería. Es decir, las variables estudiadas sí tienen una correlación. Considerando los resultados, el tipo de relación entre las variables estudiadas dependerá de otra variable: "información y práctica sobre comunicación no verbal", sobre todo respecto a la expresión e interpretación de las emociones en el rostro.

Cuando los estudiantes de ingeniería no poseen conocimientos de comunicación no verbal hay una correlación negativa débil o relación inversa poco significativa (-0.17) entre la capacidad de decodificar emociones y el rendimiento académico. Es decir, los universitarios más estudiosos, de mayor promedio, tienden a ser menos competentes para reconocer emociones. Después de recibir lecciones de comunicación no verbal, la relación entre la capacidad de reconocer emociones y el rendimiento académico se vuelve positiva débil (0.16). En otras palabras, a mayor promedio mayor capacidad para el reconocimiento de emociones.

Lo mismo ocurre con la codificación. Cuando los estudiantes han recibido información acerca de la comunicación no verbal, la capacidad de codificar facialmente las emociones y el rendimiento tienen una relación positiva muy débil (0.07).

Estos resultados obtenidos son importantes, ya que demuestran que aquellos estudiantes que han recibido 
información sobre la expresión y el reconocimiento de emociones pueden mejorar su competencia comunicativa no verbal. Esto, plantea una tarea a las instituciones educativas de todos los niveles, pero, sobre todo, a las universidades de ingeniería del Perú: incluir en sus planes de estudio un curso de comunicación no verbal. Las lecciones de comunicación no verbal deben realizarse de manera completa. Esto mejora el desempeño no verbal.

\section{Literatura citada}

Baró, Teresa (2012). La gran guía del lenguaje no verbal. Cómo aplicarlo en nuestras relaciones para lograr el éxito y la felicidad. Espasa Libros. Primera edición. España.

Castillo, Hilario, Mario (2012). Facilidad de expresión y reconocimiento faciales de emociones en jóvenes universitarios. Anales Científicos. UNALM.

Ekman, Paul (2012). El rostro de las emociones. Signos que revelan significado más allá de las palabras. Trad. Jordi Joan Serra. Ediciones RBA. Segunda edición. Barcelona.

García Pérez, Alan (2012). Pida la palabra. Por la libertad, la plenitud y el éxito. Primera edición. Titanium Editores. Lima.

Gardner, Howard (1995). Las inteligencias múltiples. Estructura de la mente. Segunda edición en español. Fondo de Cultura Económica. México.

Hernaández Sampieri, Roberto., Fernández-Collado, Carlos y Baptista Lucio, Pilar (2006). Metodología de la investigación. México. Cuarta edición. McGraw-Hill.

Knapp, Mark (1985). La comunicación no verbal. El cuerpo y el entorno. Primera reimpresión de la Primera edición en castellano. Editorial Paidós. Barcelona. 373 pp.

Punset, Eduardo (2014). El alma está en el cerebro. Radiografía de la máquina de pensar. Ediciones Destino. Primera edición. Barcelona.

(2012). Lo que nos pasa por dentro. Un millón de vidas al descubierto. Ediciones Destino. Primera edición. Barcelona. 